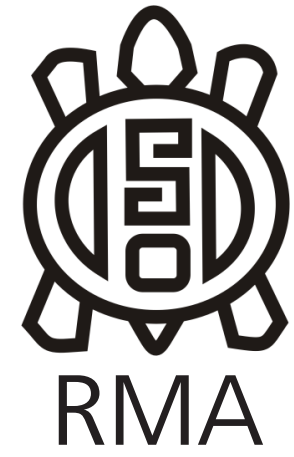

Dossier

\title{
Al pie del cerro Fitz Roy: cazadores recolectores en el Noroeste del lago Viedma, provincia de Santa Cruz
}

\author{
At the foot of Mount Fitz Roy: hunter-gatherers in the Northwest of \\ Lake Viedma, Santa Cruz province
}

\author{
Juan Bautista Belardi*, Silvana Laura Espinosa**, \\ Flavia Carballo Marina*** y Rocío Vanesa Blanco****
}

*Instituto de Ciencias del Ambiente, Sustentabilidad y Recursos Naturales - Universidad Nacional de la Patagonia Austral (Unidad Académica Río Gallegos), CONICET - Centro de Investigaciones y Transferencia de Santa Cruz, Universidad Tecnológica Nacional, Universidad Nacional de la Patagonia Austral. Email: juanbautistabelardi@gmail.com

**CONICET - Centro de Investigaciones y Transferencia de Santa Cruz, Universidad Tecnológica Nacional, Universidad Nacional de la Patagonia Austral. Email: silespinosa@gmail.com

***Universidad Nacional de la Patagonia Austral (Unidad Académica Río Gallegos) - Instituto de Ciencias del Ambiente, Sustentabilidad y Recursos Naturales. Email: flaviacarballomarina@gmail.com

****Administración de Parques Nacionales - Dirección Regional Patagonia Austral. Email: rvblanco@apn.gob.ar

\begin{abstract}
Resumen
Se presentan los resultados de nuevos trabajos en el bosque de Nothofagus en la localidad de El Chaltén, lindante con el Campo de Hielo Patagónico Sur. El ambiente es marcadamente estacional y el espacio configura un callejón sin salida. Las excavaciones de sitios en bloques La Lagunita-La Fisura, Alero 1 y Alero 2 brindan información sobre tecnología lítica y arqueofaunas que fue complementada con relevamientos de motivos rupestres y el de hallazgos aislados. El registro arqueológico muestra baja intensidad de uso y el desarrollo de actividades focalizadas en el mantenimiento de artefactos, mientras que la arqueofauna evidencia el aprovechamiento de médula. Los tres sitios poseen motivos rupestres zoomorfos que no sugieren diferencias importantes en sus momentos de ejecución. Se propone el uso marginal del bosque por parte de partidas logísticas con individuos equipados. Dos dataciones radiocarbónicas circa 400 años AP muestran la integración tardía de este ambiente a los sistemas cazadores recolectores de la margen Norte del lago Viedma. Una primera comparación en torno al uso del bosque en la margen Sur del lago y en cuencas vecinas (lagos San Martín y Argentino) indicaría la existencia de distintos umbrales de marginalidad.
\end{abstract}

Palabras clave: Lago Viedma; Bosque; Tecnología; Pinturas; Marginalidad.

\begin{abstract}
New results obtained in the Nothofagus forest around El Chaltén town, close to the Southern Patagonian Ice Field, are introduced. The environment is highly seasonal and the landscape configures a dead end. Excavations at shelters in boulders -La Lagunita-La Fisura, Alero 1 and Alero 2 archaeological sites-provide information on lithic technology and archaeofauna that was complemented by rock art surveys and the recording of isolated finds. The archaeological record shows low intensity use, activities related to artifact maintenance and the archaeofauna evidences marrow consumption. All sites have zoomorphic rock art motifs that do not suggest marked differences in their execution time. The marginal and logistic use of the forest by hunter gatherers supplied coming from the Eastern steppes is proposed. Two radiocarbon dates circa 400 years BP show the late integration of this environment within huntergatherer systems of the Lake Viedma Northern shore. An initial comparison on forest use with the Southern margin of the lake and neighboring basins (Lakes San Martín and Argentino) points to different marginality thresholds.
\end{abstract}

Keywords: Lake Viedma; Forest; Technology; Rock paintings; Marginality. 


\section{Introducción}

En forma reciente la cuenca del lago Viedma ha comenzado a ser incorporada a las investigaciones sobre el poblamiento humano del Suroeste de Patagonia. Los trabajos se han concentrado sobre la parte Noreste estepa- de la margen Norte del lago (Belardi et al., 2016, 2017, 2019a). El Oeste forma parte del Parque Nacional Los Glaciares y se caracteriza por la presencia del bosque de Nothofagus y de agujas y cerros graníticos entre los que se destacan las figuras del Torre $(3128 \mathrm{msnm})$ y el Fitz Roy (3375 msnm), en el borde Este del Campo de Hielo Patagónico Sur (CHPS) (Kosmal \& Miranda, 2008) (Figura 1). Si bien las investigaciones en el bosque, focalizadas en torno a la localidad de El Chaltén -desde donde se accede a estos grandiosos cerros-, fueron las primeras en llevarse a cabo (Belardi \& Caracotche, 2005), es en esta oportunidad que se integran a la discusión mayor sobre el uso del espacio por parte de poblaciones cazadoras recolectoras en la margen Norte del lago Viedma (Figura 1). De esta manera, se presentan los resultados de trabajos en la localidad de El Chaltén, en el ecotono del bosque de lenga (Nothofagus pumilio) y ñire (Nothofagus antarctica). Se realizaron excavaciones acotadas que proveyeron información cronológica sobre las ocupaciones humanas, tecnología lítica, arqueofauna y además se llevaron a cabo relevamientos rupestres. Los trabajos se concentraron en sitios en bloques Alero 1 y Alero 2 (Belardi \& Caracotche, 2005) y en otro sitio en bloque denominado La Lagunita-La Fisura que está constituido por dos reparos. También se da a conocer un nuevo hallazgo aislado en cercanías de la laguna Torre y se amplía la información sobre un contexto en superficie y a cielo abierto cercano al Chorrillo del Salto.

Los resultados se evalúan en la escala local en términos de la variabilidad del registro arqueológico del bosque en El Chaltén. Localizado entre los valles de los ríos Fitz Roy y De Las Vueltas, ambos alimentados por el CHPS que impide la circulación hacia la vertiente pacífica, es un lugar que conforma un claro callejón sin salida. Se destaca que el acceso a la localidad se hace por un estrecho pórtico ocupado mayormente por el curso del río De Las Vueltas (Figura 2). Además, la cercanía al campo de hielo hace que el ambiente esté expuesto a las oscilaciones relacionadas con la dinámica glaciaria, lo que refuerza la marcada estacionalidad. Estas consideraciones hacen que el concepto de marginalidad, entendido como el uso poco intenso por parte de las poblaciones cazadoras recolectoras respecto de los espacios de estepa

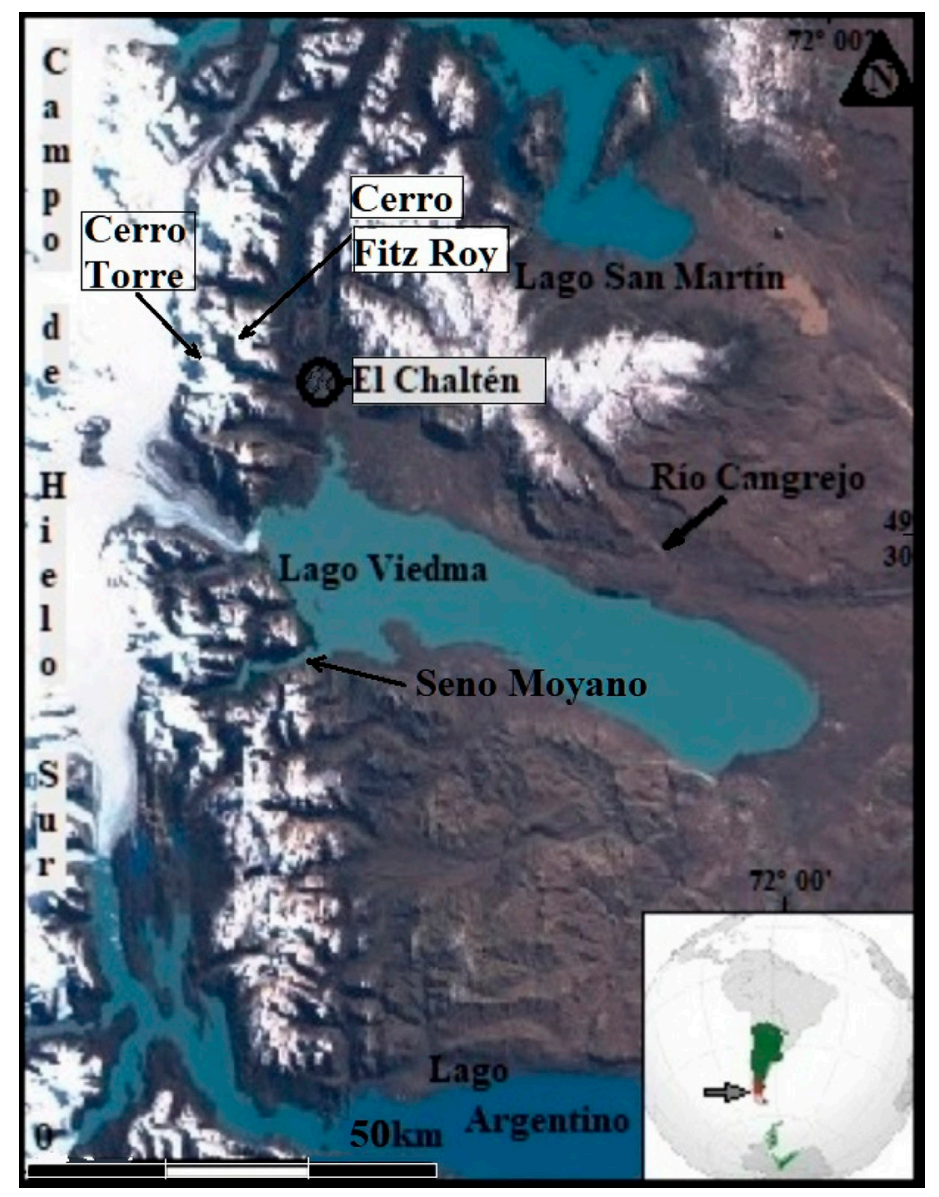

Figura 1. Mapa general de la región

Figure 1. Map of the studied region 
localizados hacia el Este (Borrero, 2004), sea central a la hora de discutir la integración del registro arqueológico del bosque con el de la estepa. Para finalizar, se ponderan los resultados en la escala regional que comprende a la margen Norte del lago Viedma, y se realiza una primera comparación sobre el uso del bosque en su margen Sur y en las cuencas lindantes de los lagos San Martín y Argentino (Figura 1).

\section{Marco ambiental y arqueológico regional}

El lago Viedma (250 msnm) ocupa un amplio valle con un marcado eje Oeste-Este, resultado de la dinámica glacial durante el Cuaternario. Tiene unos $78 \mathrm{~km}$ de longitud y sus cabeceras están conectadas al CHPS. La margen Noreste del lago está enmarcada por mesetas basálticas miocénicas (900-1100 msnm); sin embargo, al Oeste del río Cangrejo la meseta da lugar a un relieve de montaña que dificulta la circulación hacia el Norte (Figura 1). Así, se configura un amplio callejón en campos de invernada (por debajo de los 400 msnm) que conecta la estepa con el bosque de Nothofagus (Belardi et al., 2019b).

Se registraron avances neoglaciares a lo largo de los últimos 4500 años (Wenzens \& Wenzens, 1998) que generaron una mayor estacionalidad en el extremo occidental del lago (Kaplan et al., 2016; Strelin et al., 2014). Esto probablemente causó el uso intermitente o el abandono de este sector de la cuenca. Los estudios de sedimentos del Lago del Desierto señalan que alrededor de 850 años cal. D.C. comenzó la Anomalía Climática Medieval (ACM), con una duración aproximada de 600 años (Kastner et al., 2010). Luego, durante la Pequeña Edad de Hielo (PEH), el ambiente se tornó más frío y húmedo y se habría producido una expansión hacia el Este del bosque de Nothofagus (Bamonte, 2012); el periodo finalizó alrededor de 1750 D.C. (Masiokas et al., 2009).

Los trabajos arqueológicos siguieron una perspectiva distribucional que mostraron que las mayores densidades artefactuales se concentran en la estepa, en los campos bajos sobre la estrecha franja de costa ( $\leq 400 \mathrm{msnm}$ ), en los campos altos (401-900 msnm) y en la meseta basáltica ( $\geq 900 \mathrm{msnm}$ ). Las primeras evidencias de ocupaciones humanas fueron registradas en el sitio Punta del Lago 1, ubicado sobre un paredón de areniscas (500 msnm) en un estrecho cañadón al Noreste del lago. Comienzan en torno a los 3670 años AP hasta 970 años AP; se trata de ocupaciones de baja intensidad en un sector donde se destacan las manifestaciones rupestres, principalmente grabados, aunque hay unos pocos motivos pintados un guanaco y un antropomorfo- que sugieren también ocupaciones del Holoceno temprano ${ }^{1}$ (Belardi et al., 2019a). En este sentido, en las cuencas de los lagos

\footnotetext{
1 En Belardi et al. (2019a, Figura 4 A y B) se informó acerca de dos guanacos pintados. Un nuevo análisis del panel permite modificar la asignación del zoomorfo superior, no ya como guanaco, sino como un felino.
}

Tar-San Martín y Argentino -ubicadas inmediatamente al Norte y al Sur, respectivamente-, se han obtenido dataciones circa 9700 años AP, por lo que sería esperable que la cuenca del lago Viedma también mostrara una profundidad temporal semejante. En las cercanías del cañadón, un poco más hacia el Este, se dataron dos contextos a cielo abierto que muestran ocupaciones durante momentos históricos: $382 \pm 42$ años AP y 334 \pm 42 años $A P$, respectivamente (Belardi et al., 2016) que se continúan en la reserva Tehuelche-Aonikenk-Mapuche del lago Viedma (Nuevo Delaunay et al., 2020). En hoyadas de deflación con bosquetes de molle (Schinus sp.) lindantes con la costa del lago se dataron contextos arqueológicos -entre 2090 años AP y 1790 años AP- que tienen una alta densidad y diversidad artefactual (Belardi et al., 2018). El otro espacio de la margen Norte del lago Viedma que muestra también una importante señal de uso es la meseta del Tobiano, que presenta reparos basálticos con grabados rupestres y una alta frecuencia de parapetos (Belardi et al., 2017). En todos los lugares trabajados se emplearon rocas de disponibilidad local, principalmente basaltos y dacitas, con la excepción de la obsidiana proveniente de la Pampa del Asador (Espinosa \& Goñi 1999). Esta cantera se ubica aproximadamente unos $250 \mathrm{~km}$ al Noreste, considerando el trayecto directo desde el bosque hacia la desembocadura del río Cangrejo (Figura 1), girando al Norte y atravesando la laguna del Pajonal, la margen Este del lago Tar, las mesetas de San Adolfo y Cardiel Chico y las márgenes Oeste de los lagos Cardiel y Strobel.

Los antecedentes respecto del uso del bosque y sectores aledaños fueron presentados por Belardi y Caracotche (2005). Se realizaron trabajos distribucionales en torno a la bahía Túnel, inmediatamente al Este del borde de bosque actual. Los resultados de transectas y sondeos mostraron una relativa continuidad de artefactos líticos - desechos de talla en dacita, basalto, calcedonia y obsidiana, seguidos por raspadores y raederas en esas mismas materias primas- que se encontraban por encima de un depósito de ceniza, que se vincularía con la erupción del volcán Aguilera ocurrida hace unos 3300 años (Stern, 1990, 2008).

En el bosque se habían relevado sumariamente dos bloques con manifestaciones rupestres, los que son motivo ahora de un más profundo análisis: Alero $1 \mathrm{y}$ Alero 2. También se informó una baja frecuencia de materiales aislados en una hoyada de deflación cercana al Chorrillo del Salto -una baja frecuencia de lascas de dacita y basalto- y un raspador de dacita de grandes dimensiones (57 x $50 \times 16 \mathrm{~mm}$ ) sobre la senda que conduce a la laguna Torre (Figura 2), que difiere de los encontrados en bahía Túnel.

\section{Metodología}

La identificación de los sitios Alero 1 y Alero 2 y de los 
materiales aislados informados en Belardi y Caracotche (2005) se produjo a través de información provista por personal de la Administración de Parques Nacionales. Las bajas condiciones de visibilidad arqueológica que otorga el bosque (Borrero \& Muñoz, 1999) hacen que los bloques sean objetivos centrales de relevamiento; así se produjo el reconocimiento del sitio La Lagunita-La Fisura. También se relevaron senderos y espacios erosionados cercanos a los bloques pero sin registrar hallazgos.

En los bloques se realizaron sondeos y relevamientos de los motivos rupestres. El estudio de los materiales líticos se inició reconociendo sobre base macroscópica las materias primas empleadas, se estableció el estado de las piezas, sus dimensiones (largo, ancho y espesor) y se estudiaron siguiendo el procedimiento de análisis tecnotipológico propuesto por C. Aschero $(1975,1983)$. Fueron contabilizados todos aquellos desechos de talla con talón, núcleos y artefactos formatizados. Estos últimos, aún fracturados y de no haberse podido realizar un remontaje, se consideraron como una unidad. Con respecto a los restos arqueofaunísticos, se identificó especie, elemento, estadio de meteorización (Behrensmeyer, 1978), la presencia de modificaciones antrópicas y, dado el estado de fragmentación de la muestra, el largo de las astillas (entre otros, Mengoni Goñalons, 1999). Los diversos motivos rupestres fueron clasificados como figurativos, no figurativos y no determinados, en el sentido de Blanco (2015). En el caso de los motivos figurativos puede tratarse de antropomorfos y/o zoomorfos, sean figuras de cuerpo entero o huellas. El número de motivos por sitio se estableció a partir del relevamiento de campo combinado con el tratamiento digital de las fotografías mediante el uso del complemento D-Stretch, del programa J-Image (Acevedo \& Franco 2012).

\section{Resultados}

\section{Bloques}

Los tres bloques identificados como sitios arqueológicos son de granito del Complejo plutónico Fitz Roy. Son de grandes dimensiones y hoy se usan para actividades de escalada deportiva que, de manera directa o indirecta, afectan la preservación de los materiales arqueológicos, principalmente de las manifestaciones rupestres. Por ejemplo, se ha constatado la presencia de magnesio empleado para lograr mayor adherencia a la roca- en el Alero 2 en torno a motivos pintados y que el tránsito de personas alrededor de los bloques puede levantar polvo que se fije a los motivos y los oblitere. Por estas razones, el trabajo también contribuye con la fundamentación de medidas de protección puntual del registro arqueológico en el ejido urbano de la localidad de El Chaltén y en su alrededor.

\section{La Lagunita-La Fisura}

El bloque tiene una superficie aproximada de $182 \mathrm{~m}^{2} \mathrm{y}$ está en un manchón de bosque en terrenos de la Reserva Natural Urbana de El Chaltén, a unos 420 msnm, en la margen Nor-Noreste de la laguna. El sitio arqueológico está conformado por dos aleros ubicados a lo largo de $12 \mathrm{~m}$ del frente Sur del bloque (Figura 2 y $3 \mathrm{~A}$ ).

\section{$\underline{\text { La Lagunita Alero } 1}$}

El alero está formado por una visera que cubre una superficie aproximada de $30 \mathrm{~m}^{2}$. En el sector medio se excavó una cuadrícula de $50 \mathrm{~cm}$ de lado, que se planteó a partir de la presencia de cuatro artefactos líticos en superficie. El sedimento es eólico y se siguieron niveles artificiales de cinco $\mathrm{cm}$, no se reconocieron divisiones naturales. Los materiales hallados corresponden a artefactos líticos. Se registraron 57, de los cuales 29 tienen talón (Tabla 1). El 38,59 \% de los artefactos se concentra entre los 20 y los $30 \mathrm{~cm}$ de profundidad. La disminución en la frecuencia artefactual es notoria a partir de los 40 $\mathrm{cm}$ hasta alcanzar los $65 \mathrm{~cm}$. Se constató que por debajo sigue un nivel, compuesto principalmente por rocas, que es arqueológicamente estéril. Esta es la superficie glaciaria a partir de la cual se comenzó a depositar por acción eólica el sedimento areno franco que contiene a los materiales arqueológicos. Se obtuvo un valor de $\mathrm{pH}$ de 6,27 en el sedimento de los niveles inferiores y de $\mathrm{pH}$

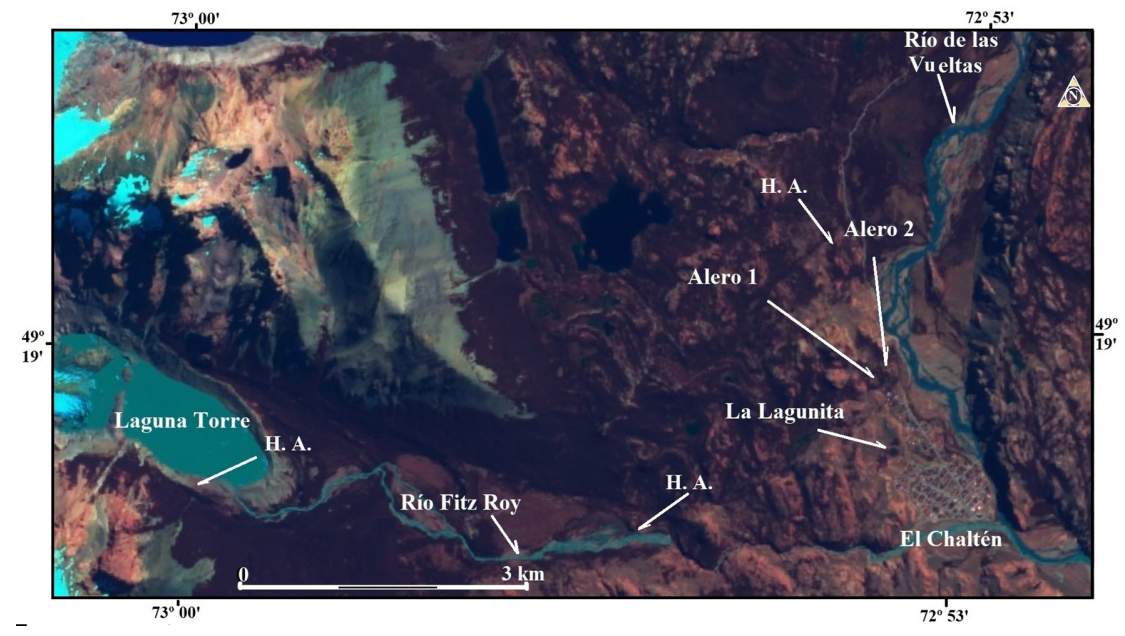

Figura 2. Ubicación de los sitios y hallazgos aislados (H. A.).

Figure 2. Location of archaeological sites and isolated findings. 
Tabla 1. Tipos artefactuales por materia prima en los sitios abordados.

Table 1. Artifactual tipes by raw material at studied sites.

Referencias: RGFO: Rocas de Grano Fino Oscuras, L. ang: lasca angular, L. indif.: lasca indiferenciada, L. sec.: lasca secundaria, L. reduc. dir: lasca de reducción directa, L. adelg.: lasca de adelgazamiento, L. red. bif.: lasca de reducción bifacial, RBO: retoque bisel oblicuo. Otras (materias primas): calcedonia, sílice, jaspe, madera silicificada, andesita, arenisca y limolita.

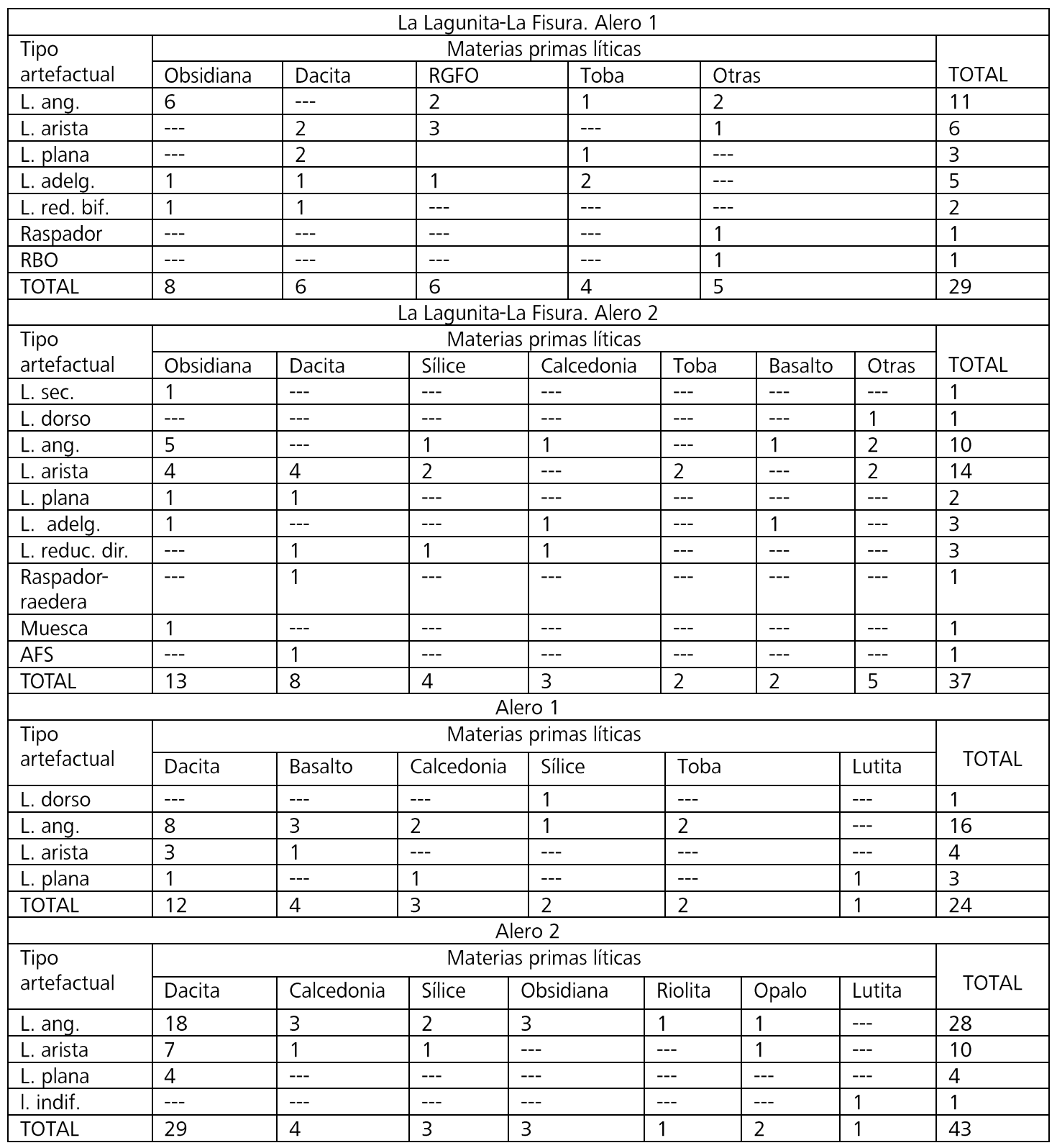

5,46 en los superiores, lo que sugiere bajas condiciones de preservación de elementos orgánicos.

Predomina el uso de la obsidiana (27,58\%), seguido por la dacita y RGFO (Rocas de Grano Fino Oscuras -Charlin, $2005-)$ en igual proporción (20,68 \%). Todas las lascas son internas; el $90 \%$ son hipermicrolascas y dominan las angulares (37,93\%) (Tabla 1). Más de la mitad de los talones son lisos (56,6\%). Se destaca la presencia de un raspador y un artefacto con retoque de bisel oblicuo. El predominio de hipermicrolascas y la presencia de lascas de adelgazamiento y de reducción bifacial evidencia actividades relacionadas con el mantenimiento de artefactos.

\section{La Lagunita Alero 2}

Este alero es en realidad una gran concavidad colmatada, formada bajo el bloque, que une sus lados Sur y Norte (Figura $3 \mathrm{~A}$ ). Sobre el techo del lado Sur de la oquedad y a 1,6 m de la boca Sur, se registraron motivos rupestres, lo que llevó a realizar bajo ellos una excavación de $1 \mathrm{~m}^{2}$. 

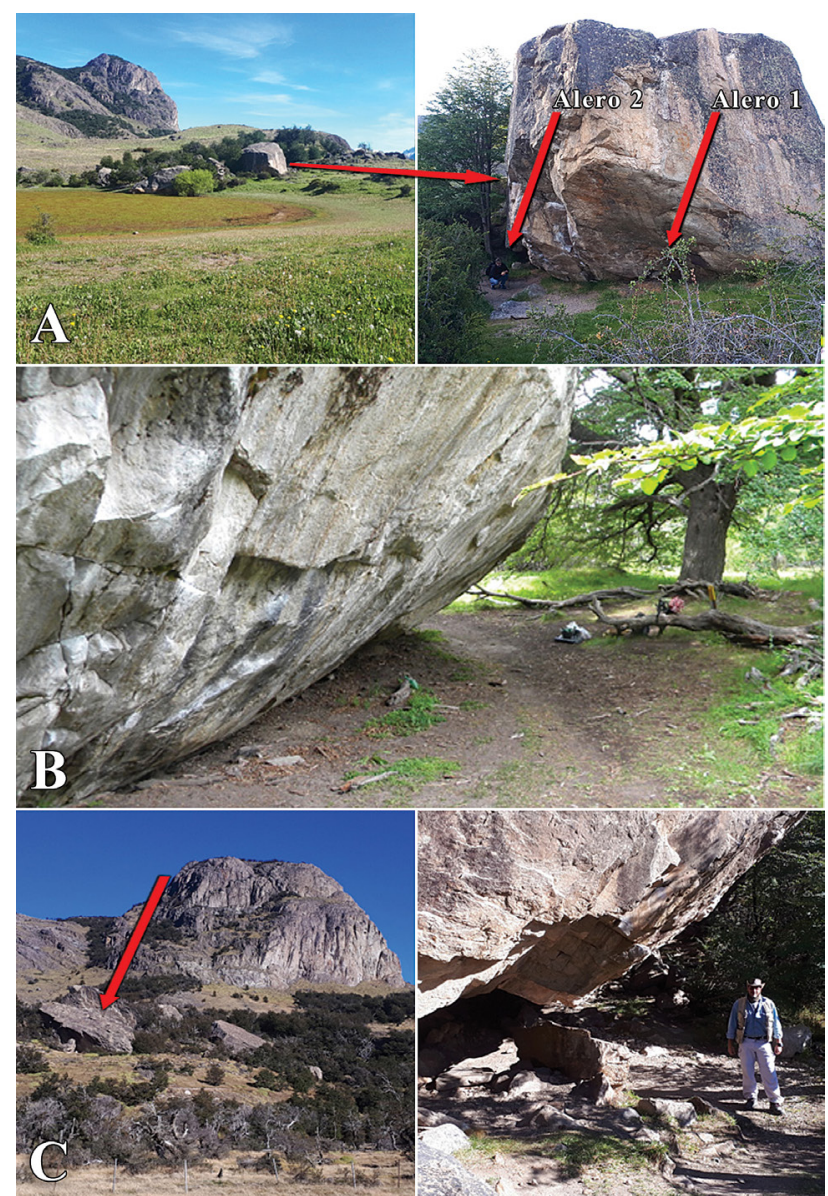

Figura 3. Vistas de los sitios estudiados.

Figure 3: Sights of archaeological sites.

Referencias: A. La Lagunita-La Fisura, B. Alero 1, C. Alero 2.

La luz entre el techo y la superficie desde donde se inició la excavación es de $55 \mathrm{~cm}$. El sedimento es eólico y tiene un nivel de ceniza de entre dos y tres $\mathrm{cm}$, a unos $10 \mathrm{~cm}$ de la superficie. Se siguieron niveles artificiales de cinco $\mathrm{cm}$. La potencia del depósito arqueológico llega hasta los $48 \mathrm{~cm}$, donde se alcanzó el nivel estéril de rocas. El relleno que contiene el contexto arqueológico es arenofranco. A diferencia del Alero 1, el menor valor de $\mathrm{pH}$ del sedimento corresponde a los niveles inferiores $(5,70)$ mientras que el de los niveles superiores es de $\mathrm{pH} 6,43$, pero las implicaciones para la potencial preservación de restos orgánicos es similar. Se registraron 72 artefactos líticos, de los cuales sólo 36 (50 \%) tienen talón (Tabla 1). El $33,33 \%$ de los artefactos se concentra entre los 18 $\mathrm{cm}$ y los $24 \mathrm{~cm}$, en tanto que en los demás niveles oscila entre cinco y nueve piezas.

Al igual que en La Lagunita Alero 1, predomina el uso de la obsidiana (35,13\%), seguido por el de la dacita $(21,62$ $\%)$. De la misma manera, si bien hay lascas externas, su representación es muy baja (5,40\%). El $95 \%$ de los desechos de talla son micro e hipermicrolascas y dominan los talones lisos (52,7\%). La mayoría de las lascas son de

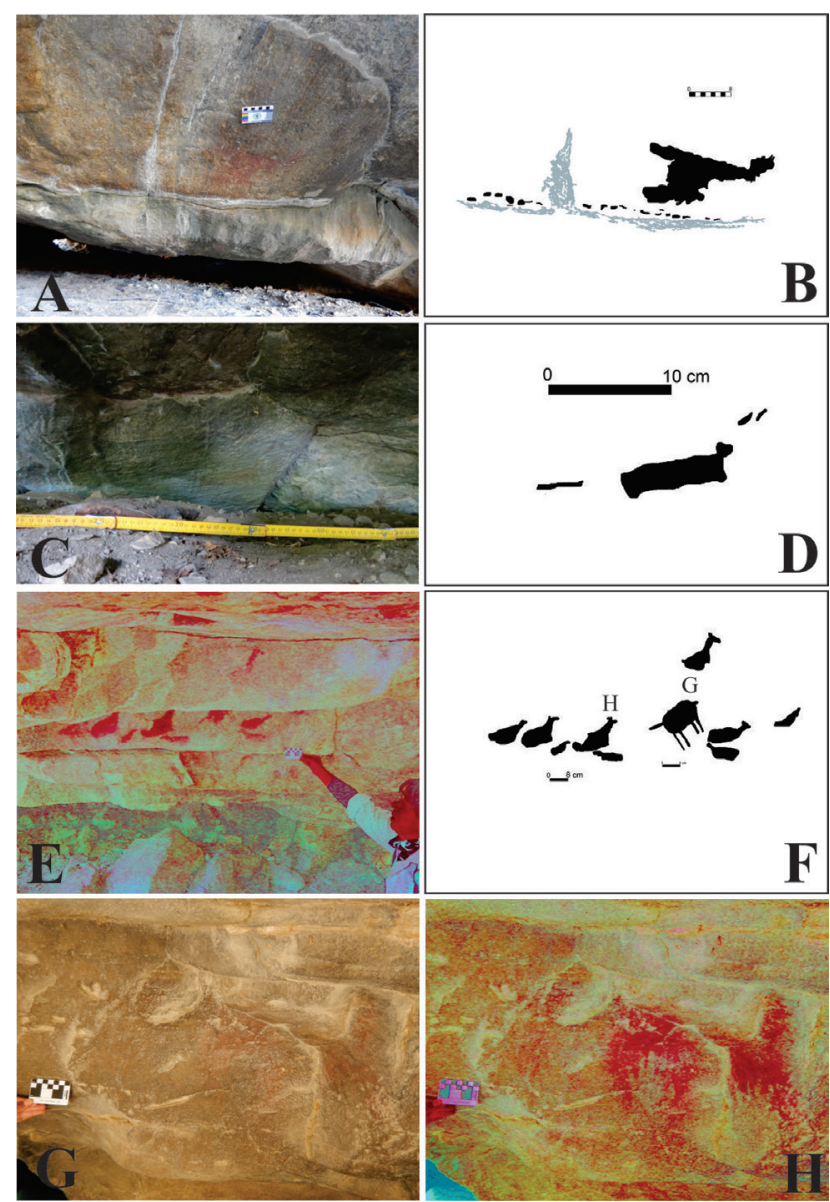

Figura 4. Motivos rupestres identificados

Figure 4. Identified rock art painted.

Referencias: A. Panel con motivos pintados en Alero 2 de Bloque La Lagunita-La Fisura. B. Calco de los motivos identificados (en gris se representan las precipitaciones de sales). C. Localización de motivos rupestres en Alero 1. D. Calco de los motivos rupestres. E. Motivos rupestres del sector 1 en Alero 2. Debajo de la escala puede observarse la mano negativa de este sector (imagen tratada digitalmente con D-Stretch, filtro Ire-15). F. Calco de motivos zoomorfos del sector 1 (G: guanaco, H: huemul). G. Sector 2 de Alero 2, con restos de pintura roja. G. Identificación de manos negativas luego de tratamiento digital de la imagen (D-Stretch, filtro Ire-15).

arista (37,83\%), seguidas por las angulares (27,02\%). Se destaca la presencia de un artefacto compuesto (raspadorraedera) y una muesca, ambos de tamaños pequeños, ya que ninguno supera los $35 \mathrm{~mm}$. Nuevamente, los tamaños de las lascas y la presencia de aquellas de adelgazamiento y reducción bifacial indican actividades relacionadas con el mantenimiento de artefactos (Tabla 1).

Se registraron tres motivos rupestres pintados en color rojo (Tabla 2 y Figura 4 A y B). El panel en el que se encuentran los motivos mide $0,8 \mathrm{~m}$ de largo por $0,75 \mathrm{~m}$ de alto y está delimitado por grietas naturales. Aquí se identificaron un zoomorfo, de $15 \mathrm{~cm}$ de alto por $22 \mathrm{~cm}$ de 
Tabla 2. Tipo y frecuencia de motivos rupestres por sitio.

Table 2. Type and frequency of rock motifs by site.

\begin{tabular}{|c|c|c|c|c|c|c|c|c|}
\hline \multirow[b]{3}{*}{ Sitio } & \multicolumn{7}{|c|}{ Tipo de motivo rupestres } & \multirow[b]{3}{*}{ TOTAL } \\
\hline & \multicolumn{4}{|c|}{ Figurativos } & \multicolumn{2}{|c|}{ No figurativos } & \multirow[b]{2}{*}{ No determinado } & \\
\hline & Zoomorfo & Guanaco & Huemul & Mano negativa & Puntiforme & Rectilíneo & & \\
\hline La Lagunita-La Fisura & 1 & & & & 1 (18 elementos) & & 1 & 3 \\
\hline Alero 1 & 1 & - & - & - & - & 2 & - & 3 \\
\hline Alero 2 & 6 & 2 & 1 & 3 & - & - & 1 & 13 \\
\hline $\begin{array}{l}\text { TOTAL } \\
\end{array}$ & 8 & 2 & 1 & 3 & 1 & 2 & 2 & 19 \\
\hline
\end{tabular}

ancho, una línea de 18 puntos, con una extensión de 38 $\mathrm{cm}$, y una superficie de pintura considerada como motivo no determinado (Tabla 2, Figura 4B). Dado que todos los motivos comparten la misma serie tonal y no se registran superposiciones, por lo que las pinturas corresponderían a un solo evento de ejecución.

\section{Alero 1}

Un kilómetro al Norte del bloque La Lagunita-La Fisura, se encuentra el Alero 1 (Figura 2). Está en medio del bosque, a $450 \mathrm{msnm}$, y dista unos $50 \mathrm{~m}$ de la senda que lleva al mirador del Cerro Fitz Roy. Tiene una superficie aproximada de $1000 \mathrm{~m}^{2}$. Su pared Sur forma un abrigo de $16,30 \mathrm{~m}$ de boca por 4,65 $\mathrm{m}$ de profundidad máxima. Hacia el extremo $E$ de esta pared hay tres motivos pintados muy desvaídos, casi al nivel del suelo actual. Al pie de los mismos hay sedimento eólico suelto, producto de la remoción por parte de un animal excavador. Allí se planteó una cuadrícula de un metro por 0,50 ms, siguiendo cinco niveles artificiales de $10 \mathrm{~cm}$ (no se reconocieron niveles naturales). Los primeros cuatro niveles presentan entre 10 y 14 artefactos y el último sólo cinco. Aquí, a los 50 $\mathrm{cm}$, además de la abrupta disminución de hallazgos el sedimento estaba muy húmedo, plástico, lo que alerta sobre problemas de preservación del registro orgánico. Se profundizó un poco más y se constató que no había potencial de enterramiento, por lo que se decidió no continuar la excavación. A lo largo de toda la estratigrafía se hallaron 50 artefactos, de los cuales el $48 \%(n=24)$ conserva el talón (Tabla 1 ). Se registraron cinco astillas óseas indeterminadas que no alcanzan los tres $\mathrm{cm}$ de longitud y unos pocos restos de roedor.

En el conjunto lítico predomina el uso de la dacita (50 $\%)$, las lascas internas y, entre ellas, las angulares $(66,66$ $\%$ ) (Tabla 1). También se registraron dos lascas sin talón de obsidiana. Todos los artefactos corresponden a microlascas, lo que sugiere que en el sitio se llevaron a cabo tareas de mantenimiento de artefactos.

Los tres motivos rupestres se encuentran en el interior de un pequeño reparo (Tabla 2 y Figura 4 C y D), a unos 50 $\mathrm{cm}$ del suelo actual, sobre un panel en posición oblicua y delimitado por grietas naturales del soporte, cuyas dimensiones son $35 \mathrm{~cm}$ de largo por $20 \mathrm{~cm}$ de alto. Se observaron dos motivos lineales (uno simple, en posición horizontal, y el otro se corresponde a dos trazos paralelos, en posición vertical), mientras que el tercer motivo fue clasificado como zoomorfo (Figura 4), mide $15 \mathrm{~cm}$ de largo por $10 \mathrm{~cm}$ de alto y se encuentra desvaído. Todos los motivos corresponden a una misma serie tonal y a un mismo momento de ejecución.

\section{Alero 2}

El sitio se encuentra a unos $200 \mathrm{~m}$ al Norte del Alero 1 (Figura 2) y a unos $60 \mathrm{~m}$ al Oeste de la senda que lleva al Chorrillo del Salto. El bloque tiene una superficie aproximada de $406 \mathrm{~m}^{2}$ y se encuentra a $427 \mathrm{msnm}$. El alero se encuentra sobre la cara Este del bloque, tiene una extensión de 11,20 $\mathrm{m}$ de boca y seis $\mathrm{m}$ de profundidad máxima. Se destaca por la presencia de tres paneles donde predominan los motivos zoomorfos. Se realizó una excavación de un $\mathrm{m}^{2}$ bajo la parte central del panel intermedio. El sedimento es eólico y se siguieron tres niveles estratigráficos artificiales de $10 \mathrm{~cm}$ cada uno, hasta llegar al nivel de rocas y rodados de base, ya que no se observaron niveles estratigráficos naturales.

Se obtuvieron dos dataciones radiocarbónicas. Una en el nivel tres (el más profundo), realizada sobre una falange de guanaco con fractura helicoidal y huellas de corte, proporcionó una antigüedad de $403 \pm 38$ años AP (AA113566) (1453-1627 cal. D.C.; 2 sigmas); mientras que la restante correspondiente al primer nivel, fue llevada a cabo sobre una apófisis de vértebra torácica de guanaco y su cronología es $343 \pm 50$ años AP (AA113565) (14591666 cal D.C.; 2 sigmas). Dado que las dataciones son estadísticamente indistinguibles al nivel de significación del 95\% (prueba estadística $\mathrm{T}=0,9127789$; chi cuadrado= 3,84; 1 grado de libertad), se considera al depósito como una unidad de formación probablemente sincrónica, con una edad radiocarbónica (media agrupada) de $381 \pm 30$ años AP (1461-1628 cal. D.C.; 2 sigmas). Las calibraciones se realizaron de acuerdo con la curva ShCal20 (Hogg et al., 2020), implementada en el programa Calib Rev.8.1.0 (Stuiver et al., 2020).

Se registraron 70 artefactos líticos, de los cuales 43 $(62,85 \%)$ conservan talón. Predomina la dacita $(67,44$ $\%)$ y las lascas angulares $(65,11 \%)$ (Tabla 1). Todos los desechos de talla son microlascas. Nuevamente, las actividades parecen haber estado relacionadas con el 
mantenimiento de artefactos. Además, se identificaron 62 restos arqueofaunísticos; 20 corresponden a guanaco (Lama guanicoe), uno a huemul (Hippocamelus bisulcus), uno a piche (Zaedyus pichiy) -placa-y 40 no pudieron ser determinados a nivel específico (se trata de fragmentos diafisiarios, cinco vértebras, dos articulaciones, una esternebra, un proximal de tibia y una primera falange).

Los 20 especímenes asignados a guanaco (se incluyen los elementos datados) corresponden a: un hioides, un fragmento de radiocúbito, tres fragmentos de primera falange (un fragmento de diáfisis, un distal con diáfisis y un proximal con diáfisis), un fragmento de diáfisis de húmero, un fragmento de metapodio proximal, un fragmento de diáfisis de fémur, un fragmento de metatarso proximal con diáfisis, dos apófisis de vértebras torácicas, dos apófisis de vértebras indeterminadas y siete fragmentos indeterminados. Los especímenes están fusionados y los 14 sobre los que se pudo establecer la meteorización (Beherensmeyer, 1978) se encuentran en estadio 1. Además de los dos elementos datados, una de las apófisis de vértebra torácica, el fragmento de metatarso y uno de los indeterminados presentan huellas de corte (25\%). Con respecto a la longitud de los fragmentos, seis son $\leq$ a $4 \mathrm{~cm}$, seis son $\leq$ a $8 \mathrm{~cm}$ y dos son $\leq$ a $9,9 \mathrm{~cm}$. La evidencia indica una relativa buena preservación de los restos óseos, el transporte de elementos axiales y apendiculares y el aprovechamiento de médula.

Hay dos sectores con motivos rupestres (Tabla 2). En el Sector 1, los motivos se disponen en tres paneles colgantes en posición vertical (Figura $4 \mathrm{E}$ ). En el panel superior, de un metro de largo por 0,25 $\mathrm{m}$ de alto, se registró un zoomorfo que se ve afectado por el uso de magnesio por parte de escaladores. En el panel intermedio, de 1,60 largo por 0,4 m de alto, se registraron nueve zoomorfos con diferente grado de preservación que se encuentran afectados por la incidencia directa del sol. Seis de ellos miran al Norte y los restantes al Sur (Figura 4 E y F). Una de las figuras que mira al Norte se asigna a una representación de huemul, ya que tiene el cuello corto en relación al tamaño del cuerpo $^{2}$, así como dos orejas pronunciadas y de forma redondeada. El motivo se encuentra a la vez afectado por un desprendimiento de la roca soporte. Otras dos figuras fueron asignadas a guanacos: sus cuellos son largos y presentan cierta proporción en relación al tamaño del cuerpo (Figura 4 F). El panel inferior $-1,10 \mathrm{~m}$ de largo por 0,25 $\mathrm{m}$ de alto-, también está afectado directamente por la luz solar. Bajo el tratamiento digital de las imágenes se identificó una mano negativa izquierda de tamaño pequeño en color rojo (Tabla 1). El Sector 2 se encuentra hacia el interior del alero: sobre un panel vertical se registraron otras

\footnotetext{
2 Aschero (2012) distingue dentro de la serie roja del estilo A3 a las representaciones de huemul, que se diferencian de las de guanaco por presentar cuellos más cortos y en las que se resalta la "cruz" (unión entre cuello y lomo). Además, las representaciones de cérvidos pueden estar intercaladas entre las tropas de guanacos.
}

dos manos negativas, también en color rojo (Figura $4 \mathrm{G}$ y $\mathrm{H}$ ). Luego del tratamiento digital de las imágenes se pudo establecer que una de ellas es izquierda, mientras que la restante tiene un bajo grado de preservación. Se distinguieron dos tonalidades de rojo: una más clara (rojo-anaranjada), que fue utilizada para la realización de los motivos zoomorfos, y otra de color más intenso, empleada para la confección de las manos negativas. Esto permite plantear al menos dos momentos de ejecución.

\section{Hallazgos a cielo abierto}

En el primer trabajo en El Chaltén (Belardi \& Caracotche, 2005) se informó de observaciones realizadas por personal de la Administración de Parques Nacionales acerca de artefactos líticos presentes en una gran cicatriz de erosión en inmediaciones del Chorrillo del Salto (valle del río De Las Vueltas) (Figura 2). Durante otra visita realizada por este personal en 2006 se observaron al menos dos raederas, una en basalto y otra de dacita, un artefacto bifacial de obsidiana y lascas y microlascas, mientras que en 2016 registraron cinco lascas: una de arista de materia prima indeterminada, otra secundaria de riolita, una de arista de sílice y las otras dos estaban enterradas. También se reconoció un raspador de sílice de unos 25 × $30 \mathrm{~mm}$. El lugar se visitó por este equipo de trabajo en febrero del año 2020 pero no se encontraron materiales. Por otra parte, se realizó un nuevo hallazgo relacionado con el valle del río Fitz Roy. Se trata de una lasca angular de obsidiana negra de $27 \times 15 \times 3 \mathrm{~mm}$ encontrada en cercanías de la margen Noreste de la laguna Torre (Figura 2).

\section{Discusión y conclusiones}

Tanto los sitios en bloques como los hallazgos aislados se ubican sobre rutas de circulación natural hacia el interior del bosque, como son los ríos Fitz Roy y Las Vueltas. Las dataciones obtenidas en el Alero 2 -circa 400 años APestán directamente relacionadas con aquellas de contextos a cielo abierto registradas en la margen Noreste del lago Viedma: $382 \pm 42$ años AP y $334 \pm 42$ años AP (Belardi et al., 2016), lo que indica el uso conjunto de la estepa y el bosque para este lapso tardío que se ubica en torno a la PEH. Las similitudes en el registro arqueológico de los otros dos sitios, así como las representaciones rupestres identificadas en el sector -que incluyen la misma serie tonal para los motivos rupestres zoomorfos-, sumado a la proximidad entre los tres bloques y ubicaciones en torno a los ríos, sugiere que también comparten este marco cronológico tardío. De todas formas, resta la posibilidad de que la producción de los motivos rupestres esté disociada temporalmente de los materiales en estratigrafía. La existencia de una mayor profundidad temporal en contextos de la estepa deja abierta la posibilidad de anteriores ocupaciones del bosque.

En los tres sitios no se registran diferencias estratigráficas que 
permitan distinguir distintos momentos de depositación: con la excepción de la obsidiana, se han empleado rocas de disponibilidad local, las frecuencias de artefactos son bajas y predominan las microlascas, entre las que se encuentran aquellas relacionadas con la reducción y el adelgazamiento de piezas, tal como sucede en La LagunitaLa Fisura. También es notoria la casi total ausencia de lascas externas y de artefactos formatizados (Tabla 1), lo que sugiere que estos artefactos debieron llegar a los sitios ya manufacturados. Allí sus filos fueron regularizados, reactivados y luego mayoritariamente descartados en otros espacios. En este sentido, se destacan los hallazgos aislados como el raspador en el sendero a la laguna Torre y el artefacto bifacial de obsidiana y el raspador del conjunto observado en la cicatriz de erosión en inmediaciones del Chorrillo del Salto. El Alero 2 se diferencia de los demás a partir de una mayor frecuencia y diversidad de motivos rupestres que presentan dos momentos de ejecución (Tabla 2). Si bien esto permite jerarquizar al sitio por sobre los otros dos, no parece indicar un uso marcadamente diferente. En este sentido, la presencia de arqueofauna -que podría responder a condiciones de preservación diferencial entre los bloques-, refuerza la propuesta de uso, ya que el valor de NISP es bajo. La caza del guanaco pudo haberse dado tanto en la estepa (a unos tres km de los bloques) como en el amplio valle del río De Las Vueltas.

Se plantea la entrada al bosque por parte de cazadoresrecolectores equipados (Kuhn, 1995) que habrían utilizado los reparos provistos por los bloques como lugares para realizar cortas estadías, llevar a cabo el mantenimiento de artefactos y plasmar motivos pintados también con baja frecuencia (Tabla 2). La escasa frecuencia de hallazgos aislados es coincidente con esta tenue señal arqueológica. Parece ser claro, entonces, que el bosque muestra ocupaciones de baja intensidad, que serían resultado de su uso logístico y que, de acuerdo con las características del área (marcada estacionalidad y constituir un "fondo de saco"), señalan su uso marginal (Borrero, 2004) con respecto a las ocupaciones de la estepa de la margen Norte del lago Viedma. Aquí las frecuencias y la diversidad de artefactos líticos, motivos rupestres, arqueofaunas y contextos de depositación son marcadamente más altas y configuran una señal arqueológica de mayor continuidad espacial y profundidad temporal, aún en las mesetas donde su uso también incluyó componentes logísticos (Belardi et al., 2017, 2019b).

Los motivos zoomorfos (Tabla 2) fueron considerados genéricamente, ya que son de tipo esquemático (sensu Gradin, 2001). Tienen cuerpo ovalado, vientre redondeado y generalmente carecen de extremidades. El cuello es corto y la cabeza, apenas esbozada, aparece muchas veces sin orejas. Los únicos tres motivos que pudieron ser referidos a nivel específico se encuentran en el Sector 1 del Alero 2 (Figura 4 F). Las representaciones zoomorfas más cercanas -guanacos- se reconocieron en el sector Noreste del lago Viedma, en la estepa. Los registrados en la meseta del Tobiano son grabados, con cuerpos rectangulares, ovales o amigdaloides con vientres abultados, con dos extremidades y cuello hacia adelante, los que se corresponderían con la variabilidad identificada por Re (2017) en el Grupo de Diseño 1. Por otro lado, el guanaco pintado de Punta del Lago 1 tiene características similares a los de la meseta. Todos estos guanacos tienen diseños diferentes a los reconocidos en el Alero 2 y a los demás motivos determinados como zoomorfos. Hacia el Sur de la cuenca, en lago Argentino, también en sitios en bloques se reconocieron motivos zoomorfos (Belardi et al., 1993; Franco et al., 1999), entre los que se identificaron huemules que presentan cornamenta u orejas alargadas -muy desarrolladas-, aunque en general tienen cuello y extremidades cortos y cuerpo redondeado (Blanco \& Caracotche, en preparación). Así, de la misma manera que en el caso de los guanacos, se observan diferencias en la representación de huemules en el ambiente de bosque de dos cuencas lacustres lindantes. En términos suprarregionales, lo que sí parece ser constante es que en el bosque las representaciones rupestres son siempre pintadas. Si bien esto puede relacionarse con una oferta disímil de soportes, tal el caso de las mesetas basálticas, marca una segregación espacial entre espacios cercanos que acompaña distintas formas e intensidades de uso por parte de las poblaciones cazadoras (Belardi \& Goñi, 2002).

A las mencionadas diferencias en las representaciones rupestres observadas en una escala espacial mayor se suman otras relacionadas con el uso del espacio del bosque. Por ejemplo, recientes trabajos en torno a la parte Sur del seno Moyano (Figura 1), margen Sur del lago Viedma, muestra una señal de intensidad aún menor que en el caso de El Chaltén. Hay un solo registro en estratigrafía, compuesto mayoritariamente por microslascas de dacita y obsidiana, recuperado en una pequeña oquedad bajo una amplia visera (Alero Simón Muniz) -sin registro de motivos rupestres- y unos pocos hallazgos aislados entre los que hay artefactos formatizados. Todo sugiere, nuevamente, baja intensidad de uso mediante una estrategia logística y actividades de mantenimiento de artefactos. No obstante, la señal arqueológica parece ser más extendida en lo espacial, ya que alcanza las cabeceras del seno. Más hacia el Sur, también se observan diferencias en frecuencias y densidades artefactuales entre, por ejemplo, los valles de los ríos Cóndor, Manga Norte, Guanaco y del arroyo Guanaco. El caso del lago Roca y el Brazo Sur, en el sector meridional del lago Argentino, también parece distinguirse. Las diferencias registradas en los motivos rupestres son acompañadas por una mayor intensidad de uso indicada por frecuencias y riqueza artefactual, distintas funcionalidades inferidas en reparos en bloques y concentraciones a cielo abierto, una continuidad relativa en la distribución de hallazgos aislados y una mayor profundidad cronológica (Borrero \& Muñoz, 1999; Franco et al., 1999). Hacia el Norte de El Chaltén, la señal de la margen Sur del lago San Martín (Belardi \& Carballo Marina, 2014) también difiere. No se registraron motivos rupestres, 
la riqueza y tamaño de los artefactos es mayor -entre los que se destacan los de obsidiana- y su distribución espacial es más extendida. Este marco de variabilidad, que se refleja en diferentes escalas espaciales y características del registro arqueológico, permite pensar en distintas condiciones y tempos de integración del bosque a los circuitos cazadores recolectores focalizados en la estepa $y$, de esa manera, en la existencia de distintos umbrales de marginalidad (Borrero, 2004) de los sectores occidentales.

La evidencia arqueológica recuperada bajo la vista del cerro Fitz Roy, además de ampliar el conocimiento acerca del uso cazador en la región y asi del patrimonio cultural de la comunidad de El Chaltén, ayuda a precisar los términos de la discusión en torno al uso del bosque en el pasado.

Río Gallegos, 15 de junio de 2020.

\section{Agradecimientos}

A la Administración Nacional de Parques Nacionales, Parque Nacional Los Glaciares, y a los Sres. Guardaparques Carlos Zoratti, Pablo Collavino, Pablo Sugliano y Simón Muniz por su inestimable colaboración. Sin la ayuda de los guardaparques el trabajo hubiese sido imposible. A la Lic. Soledad Caracotche por el registro de materiales. A la familia Viamonte (Centro Andino El Chaltén) por la información provista.

A los Dres. Juan Carlos Castro, Luis Horta, Gustavo Martínez, a la Sra. Flavia Raffo y al Sr. Pablo Binaghi por su gran ayuda en el campo. A la Lic. Patricia Campan por haber realizado el análisis arqueofaunístico de base, al Dr. Alberto Pérez por su acertado comentario sobre las representaciones rupestres y al Dr. Gustavo Barrientos por su ayuda con las calibraciones de las dataciones. Al Dr. Luis Borrero y a los dos evaluadores anónimos por la lectura del manuscrito y sus valiosas sugerencias.

Las investigaciones se realizan con proyectos UNPA y ASETUR.

\section{Bibliografía}

Acevedo, A. \& Franco, N. (2012). Aplicación de DStretchImageJ a imágenes digitales del arte rupestre de Patagonia (Argentina). Comechingonia Virtual. Revista Electrónica de Arqueología, VI(2), 152-175.

Aschero, C. (1975). Ensayo para una clasificación morfológica de los instrumentos líticos aplicada a estudios tipológicos comparativos. Informe al Consejo Nacional de Investigaciones Científicas y Técnicas.

Aschero, C. (1983). Ensayo para una clasificación morfológica de los instrumentos líticos aplicada a estudios tipológicos comparativos. Apéndices A-C revisión. Informe de la Cátedra de Ergología y Tecnología,
Facultad de Filosofía y Letras, Universidad de Buenos Aires.

Aschero, C. (2012). Las escenas de caza en Cueva de las Manos: Una perspectiva regional (Santa Cruz, Argentina). En L'art pléistocène dans le monde, Actes du Congrès IFRAO, Tarascon-sur-Ariège, septembre 2010, Symposium «Art pléistocène dans les Amériques ». $\mathrm{N}^{\circ}$ spécial de Préhistoire, Art et Sociétés, Bulletin de la Société Préhistorique, $L X V-L X V I, 807-824$. Société Préhistorique.

Bamonte, F. (2012). Cambios paleoecológicos y su posible relación con las ocupaciones humanas durante el Holoceno en el SO de Santa Cruz, Argentina [Tesis doctoral inédita]. Universidad Nacional de Mar del Plata.

Behrensmeyer, A. (1978). Taphonomic and ecologic information from bone weathering. Paleobiology, 4(2), 150-162.

Belardi, J. B. \& Caracotche, S. (2005). Resultados Arqueológicos en el Noroeste del lago Viedma, Provincia de Santa Cruz. En E. Berberián (Ed.), Actas del XIII Congreso Nacional de Arqueología Argentina (pp.109120). Universidad Nacional de Córdoba.

Belardi, J. B. \& Carballo Marina, F. (2014). La señal arqueológica del interior del bosque en la margen sur del lago San Martín (Provincia de Santa Cruz). Comechingonia, 18(2), 181-202.

Belardi, J. B., Carballo Marina, F., Barrientos, G. \& Campan, P. (2018). Southern Patagonian hunter-gatherers: Distributional archaeology in the North Shore of the lake Viedma (Santa Cruz, Argentina). Abstracts of the $83^{\text {rd. }}$ Annual Meeting of the Society for American Archaeology, pp. 35. Washington.

Belardi, J. B, Carballo Marina, F., Barrientos, G. \& Campan, P. (2019a). Punta del Lago 1: implicaciones para la arqueología de la margen Norte del lago Viedma (Santa Cruz). En J. Gómez Otero, A. Svoboda \& A. Banegas (Comps.), Arqueología de la Patagonia: el pasado en las arenas (pp. 351-359). Instituto de Diversidad y Evolución Austral (IDEAUS) - CONICET.

Belardi, J. B, Carballo Marina, F., Borrero, L. A. \& Grima, D. (2019b). Disponibilidad de campos de invernada e intensidad de uso del espacio en cuencas lacustres del Sur de Patagonia (Santa Cruz). En J. Gómez Otero, A. Svoboda \& A. Banegas (Comps.), Arqueología de la Patagonia: el pasado en las arenas (pp. 251-258). Instituto de Diversidad y Evolución Austral (IDEAUS) CONICET.

Belardi, J. B., Carballo Marina, F., Hernández Llosas, M. I. \& Cepeda, H. (1993). Arqueología del bosque: el área 
del lago Roca (Lago Argentino, Provincia de Santa Cruz). En Actas del XI Congreso Nacional de Arqueología Argentina (Tomo II, pp. 282-284). Museo de Historia Natural de San Rafael.

Belardi, J. B., Carballo Marina, F., Madrid, P., Barrientos, G. \& Campan, P. (2017). Late Holocene guanaco (Lama guanicoe) hunting grounds in Southern Patagonia: blinds, tactics and differential landscape use. Antiquity, 91(357), 718-731.

Belardi, J. B., Espinosa, S., Carballo Marina, F., Barrientos, G., Campan, P. \& Súnico, A. (2016). Desde la meseta del Cardiel Chico a la margen Norte del lago Viedma (provincia de Santa Cruz, Argentina): nuevos datos sobre el paisaje arqueológico. En F. Mena (Ed.), Arqueología de Patagonia: de Mar a Mar (pp. 411-420). Ñire Negro - Ediciones CIEP.

Belardi, J. B. \& Goñi, R. A. (2002). Distribución espacial de motivos rupestres en la cuenca del lago Cardiel (Patagonia Argentina). Boletín SIARB, 16, 29-38.

Blanco, R.V. (2015). El arte rupestre en los macizos del Deseado y Somuncurá: la producción de grabados y pinturas entre cazadores-recolectores desde el Holoceno medio [Tesis doctoral inédita]. Universidad Nacional de La Plata, La Plata.

Blanco, R. V. \& Caracotche, S. (2020). El arte rupestre del Lago Roca (Parque Nacional Los Glaciares): revisión de sitios identificados previamente [Manuscrito en preparación]. Administración de Parques Nacionales.

Borrero, L. A. (2004). The archaeozoology of Andean "Dead Ends" in Patagonia: living near the Continental Ice Cap. En M. Mondini, A. S. Muñoz \& S. Wickler (Eds.), Colonisation, Migration, and Marginal Areas. A Zooarchaeological Approach (pp. 55-61). Oxbow BooksOxford.

Borrero, L. A. \& Muñoz, A. S. (1999). Tafonomía en el bosque patagónico. Implicaciones para el estudio de su explotación y uso por poblaciones humanas de cazadores-recolectores. En Soplando en el viento. Actas de las III Jornadas de Arqueología de la Patagonia (pp. 43-56). Instituto Nacional de Antropología y Pensamiento Latinoamericano - Universidad Nacional del Comahue.

Charlin, J. (2005). Utilización de materias primas líticas en el Campo Volcánico Pali Aike (Provincia de Santa Cruz, Argentina). Revista Werken, 7, 39-56.

Espinosa, S. \& Goñi, R. (1999). Viven!! Una fuente de obsidiana en la provincia de Santa Cruz. En Soplando en el viento. Actas de las III Jornadas de Arqueología de la Patagonia (pp. 177-188). Instituto Nacional de Antropología y Pensamiento Latinoamericano Universidad Nacional del Comahue.

Franco, N., Borrero, L.A., Belardi, J. B., Carballo Marina, F., Martin, F., Campan, P., Favier Dubois, C., Stadler, N., Hernández Llosas, M. I., Cepeda, H., Muñoz, A. S., Borella, F., Muñoz, F. \& Cruz, I. (1999). Arqueología del cordón Baguales y sistema lacustre al sur del lago Argentino (provincia de Santa Cruz). Proehistoria, 3, 65-86.

Gradin, C. (2001). El arte rupestre de los cazadores de guanaco de la Patagonia. En E. Berberián \& A. Nielsen (Eds.), Historia Argentina Prehispánica (Tomo 2, pp. 839874). Editorial Brujas.

Hogg, A. G., Heaton, T. J., Hua, Q., Palmer, J. G., Turney, C. S. M., Southon, J., Bayliss, A., Blackwell, P. G., Boswijk, G., Bronk Ramsey, C., Pearson, C., Petchey, F., Reimer, P., Reimer, R. \& Wacker, L. (2020). SHCal20 Southern Hemisphere Calibration, 0-55,000 YEARS Cal BP. Radiocarbon, DOI:10.1017/RDC.2020.59

Kaplan, M. R., Schaefer, J. M., Strelin, J. A., Denton, G. H., Anderson, R. F., Vandergoes, M. J., Finke, R. C., Schwartz, R., Travis, S. G., García, J. L., Martini, M. A. \& Nielsen, S. H. H. (2016). Patagonian and Southern South Atlantic view of Holocene Climate. Quaternary Science Reviews, 141, 112-125.

Kastner, S., Enters, D., Ohlendorf, C., Haberzettl, T., Kuhn, G., Lücke, A., Mayr, C., Reyss, J. L., Wastegard, S. \& Zolitschka, B. (2010). Reconstructing 2000 years of hydrological variation derived from laminated proglacial sediments of Lago del Desierto at the eastern margin of the South Patagonian Ice Field, Argentina. Global and Planetary Change, 72, 201-214.

Kosmal. A. \& Miranda, F. (2008). Cerros Fitz Roy y Torre. Desde lo profundo de la tierra. En CSIGA (Ed.), Sitios de interés geológico de la República Argentin (Tomo II, pp. 791-802). Instituto de Geología y Recursos Minerales Servicio Geológico Minero Argentino.

Masiokas, M. H., Luckman, B. H., Villalba, R., Delgado, S., Skvarka, P. \& Ripalta, A. (2009). Little Ice Age fluctuations of small glaciers in the Monte Fitz Roy and Lago del Desierto areas, South Patagonian Andes, Argentina. Palaeogeography, Palaeoclimatology, Palaeoecology, $281,351-362$.

Mengoni Goñalons, G. (1999). Cazadores de guanacos de la estepa patagónica. Sociedad Argentina de Antropología.

Nuevo Delaunay, A., Belardi, J. B. \& Carballo Marina, F. (2020). Nuevas evidencias de sitios arqueológicos Tehuelche/Aonikenk-Mapuche (siglo XX) en Santa Cruz, Patagonia (Argentina). Magallania , 48, 161-172. 
Re, A. (2017). Grabados de guanacos en la Patagonia austral. Intersecciones en Antropología, 18, 135-147.

Stern, C. (1990). Tephrochronology of Southernmost Patagonia. National Geographic Research, 6, 110-126.

Stern, C. (2008). Holocene tephrocronology record of large explosive eruptions in the southernmost Patagonian Andes. Bulletin of Volcanology, 70(4), 435-454.

Strelin, J. A., Kaplan, M. R., Vandergoes, M. J., Denton, G. H. \& Schaefer, J. M. (2014). Holocene glacier history of the Lago Argentino basin, Southern Patagonian Icefield. Quaternary Science Reviews, 101, 124-1455.

Stuiver, M., Reimer, R P. J. \& Reimer, R. W. (2020). CALIB 8.2 [WWW program] at http://calib.org, acceso 19-09-2020.

Wenzens, G. (1999). Fluctuations of Outlet and Valley Glaciers in the Southern Andes (Argentina) during the Past 13,000 Years. Quaternary Research, 51, 238-247.

Wenzens G. \& Wenzens, E. (1998). Late glacial and Holocene glacier advances in the area of lago Viedma (Patagonia, Arg.). Zentralatt Geologie und Paläontologie, I, 593-608. 